\title{
A Simplified Implicit Maxwell Solver
}

\author{
Paolo Ricci, ${ }^{*}$, Giovanni Lapenta, ${ }^{*}, \ddagger$ and J. U. Brackbill $\ddagger$ \\ * Dipartimento di Fisica, Istituto Nazionale Fisica della Materia (INFM), Corso Duca degli Abruzzi 24, 10129 \\ Torino, Italy; †Dipartimento di Energetica, Politecnico di Torino, Corso Duca degli Abruzzi 24, 10129 Torino, \\ Italy; and $\ddagger$ Theoretical Division, Los Alamos National Laboratory, Los Alamos, New Mexico 87545 \\ E-mail: lapenta@lanl.gov
}

Received March 22, 2002; revised July 23, 2002

\begin{abstract}
We apply the second-order formulation of Maxwell's equations proposed by Jiang et al. (1996, J. Comput. Phys. 125, 104) to the solution of the implicit formulation of the three-dimensional, time-dependent Vlasov-Maxwell's system. An implicit finite difference algorithm is developed to solve the Maxwell's equations in a bounded domain with physical boundary conditions comprising electrically conducting walls (perfect conductors) and constant magnetic flux walls. We formulate the boundary conditions for Maxwell's equations to satisfy Poisson's equation throughout the domain by solving it only on the boundary. This eliminates the need for a separate projection step. We compare numerical results with analytical solutions for electromagnetic waves in vacuo, and using the implicit particle-in-cell code CELESTE3D, we test the new solver on the geospace environment modeling magnetic reconnection challenge problem. (c) 2002 Elsevier Science (USA)
\end{abstract}

Key Words: Maxwell's equations; divergence constraints; boundary conditions; implicit numerical method; particle-in-cell implicit method.

\section{INTRODUCTION}

By computing the self-consistent motion of a large number of charged particles in the fields they produce, one can simulate the full complexities of a plasma from first principles $[6,25]$. Such simulations have made important contributions to magnetic confinement [55], laser fusion [30], and space plasmas [61].

This paper is about the application of the second-order formulation of Maxwell's equations proposed by Jiang et al. [32] to the solution of the full Vlasov-Maxwell system.

The numerical solution of Maxwell's equations is a topic widely discussed in the literature. The basic difficulty in solving the typical, first-order Maxwell's equations is related to the fact that Maxwell's equations comprise a system of eight equations for six unknowns (assuming charge and current densities are given). Nevertheless, it is incorrect to consider the divergence equations redundant, on the basis that the divergence conditions will be 
satisfied if they are initially, as is often done [24]. It has been shown that ignoring the divergence conditions leads to incorrect solutions of Maxwell's equations [32, 50].

The second-order formulation of Maxwell's equations has also been considered for the implementation of a numerical algorithm and there are many reports regarding numerical solutions which do not satisfy the divergence constraints [5, 49, 53, 60]. The secondorder Maxwell's equations are derived from the first-order equations by applying the curl operator: The equations so obtained admit more solutions than do their progenitors. The spurious solutions, in particular, do not satisfy the divergence laws [32]. In this context, it is shown that boundary conditions play a crucial role in satisfying the constraints on the solution of Maxwell's equations [1, 32]. Jiang et al. show that if one satisfies Gauss's law on a part of the boundary at all times, the solution of Faraday's and Ampère's laws will satisfy Gauss's law throughout the volume [32].

Within this context, in their paper Jiang et al. describe a node-based least-squares finite element method able to achieve the satisfaction of the divergence conditions [32].

Here, we propose a finite difference algorithm, based on the second-order formulation of Maxwell's equations and its boundary conditions, as proposed by Jiang et al., applied in the context of plasma simulation using an implicit method for the solution of the VlasovMaxwell system.

Implicit simulation methods are useful for the study of low-frequency phenomena and realistic electron-ion mass ratios. Predictions of self-generated magnetic fields in laserplasma interactions [19] were confirmed by experiments in Ref. [62]. Implicit simulations of quasi-perpendicular [20] and quasi-parallel shocks [46] reveal complex behavior that is the subject of continuing study [52]. Simulations of the lower-hybrid-drift instability give estimates of the nonlinear saturation mechanism [10]. A more recent reexamination of this problem led to the discovery of the drift-kink instability [26] and the nonlinear development of the Kelvin-Helmholtz instability [37]. Results for beam filamentation and expansion into a vacuum are reported in Ref. [14]. Studies of magnetic reconnection are reported in Refs. [17, 22, 23, 37, 57]. Comparisons of implicit and hybrid solutions for switch-off shocks [13] and contact discontinuities [35, 39] help to define the range of validity of hybrid methods. The implicit moment method is also applied to plasma opening switch modeling in Ref. [43].

We remark that in the context of the plasma simulations, additional difficulties arise in solving Maxwell's equations. Implicit plasma simulations require the solution of the Maxwell equations in the presence of a permittivity in the form of a nonsymmetric tensor. Also, in the context of plasma simulations, it is pointed out that the continuity equation may be not satisfied [22]. Consistent with this observation, projection is used to assure that Poisson's equation is satisfied. (Charge-conserving algorithms have also been developed that eliminate the need to solve Poisson's equation $[18,58]$, but these have not yet been employed in implicit simulations.) In a common projection method, a scalar potential is introduced as a correction to the electric field and is solved to satisfy Gauss's law, thus bringing into correspondence the number of boundary conditions and unknowns for the electric field $[22,33]$. However, in implicit formulations, projection is only approximately correct because of a coupling of longitudinal and transverse components of the electric field by the plasma permittivity [12].

The main point of this paper is that Poisson's equation can be satisfied in the volume without the need for projection through a new formulation of the boundary conditions for Maxwell's equations following Jiang et al. [32]. 
The paper is organized as follows. First, we discuss Jiang et al.'s second-order Maxwell's equations, focusing on the boundary conditions in the context of the Vlasov-Maxwell system. These boundary conditions take an especially simple form for perfectly conducting and magnetic flux surfaces. Next, we describe the numerical implementation of consistent boundary conditions in CELESTE3D, a particle-in-cell (PIC) code based on the implicitmoment plasma simulation method [9, 59]. Finally, we present the results of calculations for electromagnetic wave propagation in vacuo and for the geospace environment modeling (GEM) magnetic reconnection challenge [7].

\section{GOVERNING EQUATIONS}

In this section, the first-order Maxwell system is stated in a spatial finite domain with physical boundaries comprising electric walls (perfect conductors) and magnetic symmetry walls. The second-order Maxwell equations are then deduced: Their formulation is discussed in order to take into account implicitly the divergence constraints.

\subsection{The First-Order Maxwell Equations}

Following Jiang et al. [32], we consider Maxwell's equations in the finite domain $\Gamma=\Omega \times I$, where $\Omega \subset \mathfrak{R}^{3}$ represents the bounded, simply connected and convex, spatial domain, while $I=[0, T]$ denotes the temporal interval of interest. (The extension to multiply connected domains is straightforward, but the presence of reentrant corners raises a number of problems which we will not consider [2].) The spatial domain $\Omega$ has a piecewise smooth boundary $\partial \Omega=\partial \Omega_{1} \cup \partial \Omega_{2}$, where $\partial \Omega_{1}$ denotes a perfect conductor and $\partial \Omega_{2}$ a magnetic flux surface. Generalizing to periodic boundary conditions is straightforward. The generic point in the integration domain is denoted by the coordinates $(\mathbf{r}, t)$.

For general, time-varying three-dimensional fields, Maxwell's equations can be stated (in Gaussian units), in the domain $\Gamma$, as

$$
\left\{\begin{array}{l}
\nabla \times \mathbf{E}+\frac{1}{c} \frac{\partial \mathbf{B}}{\partial t}=0 \\
\nabla \times \mathbf{B}-\frac{1}{c} \frac{\partial \mathbf{E}}{\partial t}=\frac{4 \pi}{c} \mathbf{J} \\
\nabla \cdot \mathbf{E}=4 \pi \rho \\
\nabla \cdot \mathbf{B}=0 .
\end{array}\right.
$$

In addition there is a charge continuity equation,

$$
\frac{\partial \rho}{\partial t}+\nabla \cdot \mathbf{J}=0
$$

and a momentum equation,

$$
\frac{\partial \mathbf{J}}{\partial t}=\frac{q}{m}[\rho \mathbf{E}+\mathbf{J} \times \mathbf{B}-\nabla \cdot \mathbf{P}]
$$

where $\mathbf{E}$ is the electric field, $\mathbf{B}$ is the magnetic flux density, $\rho$ is the charge density, $\mathbf{J}$ is the charge current density, and $\mathbf{P}$ is the pressure tensor (the pressure is assumed given for the purpose of discussion). The equations are written in microscopic form, so that the 
electric flux density coincides with the electric field, the magnetic flux density equals the magnetic field, and the dielectric permittivity and the magnetic permeability are unitary. The extension to physical systems with scalar and constant dielectric permittivity and magnetic permeability is trivial.

The initial conditions for solving Maxwell's equations are assumed to be

$$
\left\{\begin{array} { l } 
{ \mathbf { E } ( \mathbf { r } , 0 ) = \mathbf { E } ^ { 0 } ( \mathbf { r } ) } \\
{ \mathbf { B } ( \mathbf { r } , 0 ) = \mathbf { B } ^ { 0 } ( \mathbf { r } ) }
\end{array} \text { with } \left\{\begin{array}{l}
\nabla \cdot \mathbf{E}^{0}=4 \pi \rho(\mathbf{r}, 0) \\
\nabla \cdot \mathbf{B}^{0}=0
\end{array} \text { for } \mathbf{r} \in \Omega\right.\right.
$$

while the boundary conditions, on a perfectly conducting wall $\partial \Omega_{1}$, can be expressed as

$$
\left\{\begin{array}{l}
\mathbf{n} \times \mathbf{E}=0 \\
\mathbf{n} \cdot \mathbf{B}=0
\end{array}\right.
$$

and, on a constant flux wall $\partial \Omega_{2}$, as

$$
\left\{\begin{array}{l}
\mathbf{n} \cdot \mathbf{E}=0 \\
\mathbf{n} \times \mathbf{B}=0
\end{array}\right.
$$

On a constant magnetic flux surface wall, $\partial \Omega_{2}$, the boundary condition

$$
\mathbf{n} \cdot \mathbf{J}=0
$$

is respected.

\subsection{The Second-Order Maxwell Equations}

Using the div-curl method, Jiang et al. [32] show that the full first-order system of Maxwell's equations is equivalent to two decoupled systems, one involving the electric field $\mathbf{E}$ and the other involving the magnetic flux density $\mathbf{B}$.

The second-order system for the electric field $\mathbf{E}$ can be stated as

$$
\begin{cases}\nabla \times \nabla \times \mathbf{E}+\frac{1}{c^{2}} \frac{\partial^{2} \mathbf{E}}{\partial t^{2}}=-\frac{4 \pi}{c^{2}} \frac{\partial \mathbf{J}}{\partial t} & \text { in } \Gamma \\ \nabla(\nabla \cdot \mathbf{E}-4 \pi \rho)=0 & \text { in } \Gamma \\ \mathbf{n} \times \mathbf{E}=0 & \text { on } \partial \Omega_{1} \times I \\ \nabla \cdot \mathbf{E}=4 \pi \rho & \text { on } \partial \Omega_{1} \times I \\ \mathbf{n} \cdot \mathbf{E}=0 & \text { on } \partial \Omega_{2} \times I \\ \mathbf{n} \times(\nabla \times \mathbf{E})=0 & \text { on } \partial \Omega_{2} \times I,\end{cases}
$$

where the curl-curl equation is supplemented by a divergence constraint and a modified set of boundary conditions suitable for a second-order spatial equation. The initial conditions required by a temporal second-order system are the initial electric field $\mathbf{E}^{0}$ and its initial temporal derivative

$$
\left.\frac{\partial \mathbf{E}}{\partial t}\right|_{t=0}=c \nabla \times \mathbf{B}^{0}-4 \pi \mathbf{J}(\mathbf{r}, 0)
$$

which couples the electric field $\mathbf{E}$ and the magnetic flux density $\mathbf{B}$. 
It should be pointed out that system (8) is fully equivalent to the first-order Maxwell system (1) in the sense that they yield identical solutions.

A similar system for the magnetic flux can be deduced. In any case, once the electric field $\mathbf{E}$ is known from the solution of system (8), the magnetic flux $\mathbf{B}$ can be readily evaluated by integrating the first equation of system (1),

$$
\mathbf{B}(\mathbf{r}, t)=-c \int_{0}^{t} \nabla \times \mathbf{E}\left(\mathbf{r}, t^{\prime}\right) d t^{\prime}+\mathbf{B}^{0}(\mathbf{r}) .
$$

Using a least-squares method, Jiang et al. [32] prove that the second equation in system (8), the divergence equation, can be removed as it is implicitly satisfied by the curl-curl equation and the boundary conditions. A proof is also given by Mayergoyz and d'Angelo [40] when $\partial \Omega_{1} \equiv \partial \Omega$ and the mathematical details involved in the proof are discussed in Ref. [28]. To illuminate some properties of the divergence law related to the implementation of a numerical algorithm to solve Maxwell's equations, a different proof is furnished in the present paper.

The function $\chi$ is introduced as

$$
\chi=\nabla \cdot \mathbf{E}-4 \pi \rho
$$

and Gauss's law is satisfied if and only if $\chi \equiv 0$.

By applying the divergence operator to the curl-curl equation in system (8), it is possible to deduce the equation satisfied by $\chi$. Two possibilities exist. One can study the first equation of system (8) as it is, or one can develop $\nabla \times \nabla \times \mathbf{E}$ using Gauss's law. Two different equations for $\chi$ can be obtained following the two approaches. These two equations are both considered.

\subsubsection{The Equation for $\chi$ When the Straight Curl-Curl Equation is Considered}

By applying the divergence operator to the curl-curl equation in system (8), the following equation for the function $\chi$ is obtained:

$$
\frac{\partial^{2} \chi}{\partial t^{2}}=0
$$

If the initial conditions are such that

$$
\left\{\begin{array}{l}
\chi(\mathbf{r}, 0)=0 \\
\left.\frac{\partial \chi}{\partial t}\right|_{t=0}=0
\end{array} \text { for } \mathbf{r} \in \Omega\right.
$$

then $\chi \equiv 0$, Gauss's law is respected, and the divergence constraint in system (8) is implicitly satisfied. This means that system (8) can be simplified to

$$
\begin{cases}\nabla \times \nabla \times \mathbf{E}+\frac{1}{c^{2}} \frac{\partial^{2} \mathbf{E}}{\partial t^{2}}=-\frac{4 \pi}{c^{2}} \frac{\partial \mathbf{J}}{\partial t} & \text { in } \Gamma \\ \mathbf{n} \times \mathbf{E}=0 & \text { on } \partial \Omega_{1} \times I \\ \nabla \cdot \mathbf{E}=4 \pi \rho & \text { on } \partial \Omega_{1} \times I \\ \mathbf{n} \cdot \mathbf{E}=0 & \text { on } \partial \Omega_{2} \times I \\ \mathbf{n} \times(\nabla \times \mathbf{E})=0 & \text { on } \partial \Omega_{2} \times I .\end{cases}
$$


In view of developing a numerical algorithm to solve system (14), one can ask what the properties of the solution (14) will be if the initial conditions given by Eq. (13) for $\chi$ are not satisfied. Consider a perturbation, expressed in Fourier harmonics, with $\chi$ written as

$$
\chi(\mathbf{r}, t)=\sum_{\mathbf{k}} \chi_{\mathbf{k}}(t) \exp (i \mathbf{k} \cdot \mathbf{r})
$$

and initial conditions

$$
\chi_{\mathbf{k}}(0)=A_{\mathbf{k}},\left.\quad \frac{d \chi_{\mathbf{k}}(t)}{d t}\right|_{t=0}=B_{\mathbf{k}} .
$$

Because $\chi$ satisfies Eq. (12),

$$
\chi_{\mathbf{k}}(t)=B_{\mathbf{k}} t+A_{\mathbf{k}},
$$

and, as a consequence, an initial perturbation grows linearly in time.

\subsubsection{The Equation for $\chi$ Developing the Curl-Curl Equation Using Gauss's Law}

Using the vector identity

$$
\nabla \times \nabla \times \mathbf{E}=\nabla(\nabla \cdot \mathbf{E})-\nabla^{2} \mathbf{E}
$$

and the Gauss equation to evaluate $\nabla \cdot \mathbf{E}$, one can write the curl-curl equation as

$$
\nabla^{2} \mathbf{E}-\frac{1}{c^{2}} \frac{\partial^{2} \mathbf{E}}{\partial t^{2}}=\frac{4 \pi}{c^{2}} \frac{\partial \mathbf{J}}{\partial t}+4 \pi \nabla \rho
$$

To study the divergence constraint, the divergence operator is applied to Eq. (19), and the function $\chi$ is shown to satisfy the wave equation

$$
\frac{1}{c^{2}} \frac{\partial^{2} \chi}{\partial t^{2}}=\nabla^{2} \chi
$$

where the boundary conditions for $\chi$ can be derived from system (8).

In fact, regarding $\partial \Omega_{1}$, the boundary condition for Eq. (20) can be expressed simply as $\chi=0$. On $\partial \Omega_{2}$, the normal component of the gradient of the function $\nabla \cdot \mathbf{E}$ can be considered,

$$
[\nabla(\nabla \cdot \mathbf{E})] \cdot \mathbf{n}=\left[\nabla^{2} \mathbf{E}+\nabla \times \nabla \times \mathbf{E}\right] \cdot \mathbf{n}=\nabla^{2} \mathbf{E} \cdot \mathbf{n},
$$

where we have observed that $(\nabla \times \nabla \times \mathbf{E}) \cdot \mathbf{n}=0$, as follows from the boundary condition on $\partial \Omega_{2}, \mathbf{n} \times(\nabla \times \mathbf{E})=0$ [Eq. (8)]. Noting the boundary conditions $\mathbf{n} \cdot \mathbf{E}=0$ and $\mathbf{J} \cdot \mathbf{n}=0$ on $\partial \Omega_{2}$ expressed by Eqs. (8) and (7), the projection of Eq. (19) along $\mathbf{n}$ leads to

$$
\nabla^{2} \mathbf{E} \cdot \mathbf{n}-4 \pi \nabla \rho \cdot \mathbf{n}=0,
$$

and, remembering Eq. (21), it follows that

$$
\nabla(\nabla \cdot \mathbf{E}-4 \pi \rho) \cdot \mathbf{n}=\nabla \chi \cdot \mathbf{n}=0,
$$


so that the boundary conditions for Eq. (20) can be summarized as

$$
\begin{cases}\chi=0 & \text { on } \partial \Omega_{1} \times I \\ \mathbf{n} \cdot \nabla \chi=0 & \text { on } \partial \Omega_{2} \times I\end{cases}
$$

If the initial conditions are expressed as in Eq. (13), Eq. (20) with the boundary conditions given by Eq. (24) leads to $\chi \equiv 0$ : Gauss's law is respected and the divergence constraint in system (8) is implicitly satisfied. In this case, system (8) is written as

$$
\begin{cases}\nabla^{2} \mathbf{E}-\frac{1}{c^{2}} \frac{\partial^{2} \mathbf{E}}{\partial t^{2}}=\frac{4 \pi}{c^{2}} \frac{\partial \mathbf{J}}{\partial t}+4 \pi \nabla \rho & \text { in } \Gamma \\ \mathbf{n} \times \mathbf{E}=0 & \text { on } \partial \Omega_{1} \times I \\ \nabla \cdot \mathbf{E}=4 \pi \rho & \text { on } \partial \Omega_{1} \times I \\ \mathbf{n} \cdot \mathbf{E}=0 & \text { on } \partial \Omega_{2} \times I \\ \mathbf{n} \times(\nabla \times \mathbf{E})=0 & \text { on } \partial \Omega_{2} \times I .\end{cases}
$$

Following the same analysis of the previous section, it is possible to study the growth of an initial perturbation for the function $\chi$. In contrast to Eq. (17), it is found that an initial perturbation does not grow, its behavior is oscillatory, and the initial perturbation bounds $\chi$, as it is

$$
\chi_{\mathbf{k}}=\frac{i \omega A_{\mathbf{k}}+B_{\mathbf{k}}}{2 i \omega} \exp (i \omega t)+\frac{i \omega A_{\mathbf{k}}-B_{\mathbf{k}}}{2 i \omega} \exp (-i \omega t)
$$

where $\omega=c k$.

\section{THE NUMERICAL ALGORITHM}

For problems where one needs a full kinetic description of a plasma, one has no choice but to use the most general model. Mason [41] and Denavit [16] introduced implicit plasma simulation methods, which retain a full kinetic model but are much more efficient for lowfrequency plasma phenomena because they eliminate many numerical stability constraints on the time and space steps.

While the explicit methods are constrained to follow the fastest time scale of the physical system, so that the time step limit in an explicit plasma simulation is fixed by $\omega_{\mathrm{pe}} \Delta t<2$ (where $\omega_{\mathrm{pe}}$ is the electron plasma frequency), the time step constraints that have been discussed for the implicit methods are a Courant limit imposed by the propagation of acoustic waves $[9,41]$ and an accuracy limit arising from the derivation of the fluid moment equations $[9,11]$. This limit restricts the mean particle motion to one grid cell per time step, effectively

$$
v_{\text {th, } \mathrm{e}} \Delta t / \Delta x<1
$$

since the electron thermal velocity is usually larger than the ion thermal velocity. In a typical simulation of magnetospheric plasma, the time step of the implicit method can be 40 times longer than that allowed for an explicit simulation.

The grid spacing limit for the explicit method, $\Delta x<\varsigma \lambda_{\text {De }}$, where $\varsigma$ is a parameter depending on the interpolation scheme and $\lambda_{\text {De }}$ is the electron Debye length, is replaced by 
within the implicit method, and the gain in the grid spacing limit is about 10 in a typical magnetosphere simulation.

A limit on the time step imposed by the number of simulation particles is discussed in Ref. [34]. Denoting with $N_{\mathrm{pc}}$ the number of simulation particles per cell, the time step must satisfy the inequality

$$
\left(\omega_{\mathrm{pe}} \Delta t\right)^{2}<N_{\mathrm{pc}}
$$

A study of energy conservation with the direct implicit method shows that energy is conserved very accurately for $\omega_{\mathrm{pe}} \Delta t \leq 2$ and that energy errors are small even for very large time steps [14]. Results with the implicit moment method in one dimension, where stability is observed with $v_{\mathrm{th}, \mathrm{e}} \Delta t / \Delta x=0.5$ and excellent energy conservation is observed with $v_{\text {th, }, \mathrm{e}} \Delta t / \Delta x=0.01$ and $\left(\omega_{\mathrm{pe}} \Delta t\right)^{2}=5.25 \times 10^{6} \gg N_{\mathrm{pc}}=10$, suggests that Eq. (27) is a sufficient limit [59].

Mason's implicit moment method reduces the number of equations that must be solved self-consistently to a set of coupled fluid moment and field equations. The solution of these equations implicitly, together with the subsequent explicit solution of the particle equations of motion in the resulting fields, is surprisingly stable and accurate. The implicit moment method is extended to electromagnetic plasma dynamics in two dimensions in Refs. [9, 11] and in three dimensions in Ref. [56]. (Direct implicit methods, which replace the fluid moment equations by an expansion about the unperturbed particle orbits are described for electrostatic plasmas in Ref. [34], magnetized plasma in Ref. [3], and electromagnetic plasmas in Ref. [22].)

The implicit moment method formulation is described in Refs. [9, 11, 59]. Here we show how this formulation is changed when we solve the second-order Maxwell system (8) for the electric field $\mathbf{E}$, in the form given by Eqs. (14) and (25). The system is, of course, coupled to Eq. (10) for the magnetic flux density $\mathbf{B}$.

In this section, the numerical algorithm for the implicit moment method is reviewed and discretization in time and space for Maxwell's equations is described.

\subsection{Temporal Discretization}

The solution is advanced in the time domain $I$ with discrete time steps $\Delta t$, from the initial time, $t^{0}=0$, to the final time, $t^{N}=T$. Corresponding to the time step $n\left(t=t^{n}\right)$, the fields are denoted with $\mathbf{E}^{n}(\mathbf{r})$ and $\mathbf{B}^{n}(\mathbf{r})$.

The charge density $\rho^{n}$, the current density $\mathbf{J}^{n}$, and the pressure $\mathbf{P}^{n}$ are accumulated from a distribution of particles which represent the plasma. Each particle has a unique label $p$, a coordinate $\mathbf{r}_{p}$, a charge $q_{p}$, a mass $m_{p}$, and a velocity, $\mathbf{u}_{p}$. The accumulation uses shape functions, $S(\mathbf{r})$ typically b-splines, which are normalized and have bounded support, and is expressed as a summation over particles,

$$
\begin{aligned}
& \rho(\mathbf{r})=\sum_{p} q_{p} S^{(l)}\left(\mathbf{r}-\mathbf{r}_{p}\right) \\
& \mathbf{J}(\mathbf{r})=\sum_{p} q_{p} \mathbf{u}_{p} S^{(l-1)}\left(\mathbf{r}-\mathbf{r}_{p}\right) \\
& \mathbf{P}(\mathbf{r})=\sum_{p} m_{p} \mathbf{u}_{p} \mathbf{u}_{p} S^{(l-2)}\left(\mathbf{r}-\mathbf{r}_{p}\right),
\end{aligned}
$$

where the superscript $l$ indicates the order of the b-splines for each accumulation $S$, following Ref. [59]. 
Semidiscrete, continuous space approximations to Maxwell's equations are written

$$
\left\{\begin{array}{l}
\nabla \times \mathbf{E}^{n+\theta}+\frac{1}{c} \frac{\mathbf{B}^{n+1}-\mathbf{B}^{n}}{\Delta t}=0 \\
\nabla \times \mathbf{B}^{n+\theta}-\frac{1}{c} \frac{\mathbf{E}^{n+1}-\mathbf{E}^{n}}{\Delta t}=\frac{4 \pi}{c} \mathbf{J}^{n+\frac{1}{2}} \\
\nabla \cdot \mathbf{E}^{n+\theta}=4 \pi \rho^{n+\theta} \\
\nabla \cdot \mathbf{B}^{n}=\nabla \cdot \mathbf{B}^{n+1}=0,
\end{array}\right.
$$

where $\theta \in[1 / 2,1]$ is a parameter chosen to adjust the numerical dispersion relation for electromagnetic waves (for $\theta<1 / 2$, the algorithm is shown to be unstable [11]). We note that for $\theta=1 / 2$ the scheme is second-order accurate in $\Delta t$; for $1 / 2<\theta \leq 1$ the scheme is first-order accurate. Higher order differencing schemes are described in Ref. [47]. Variables at time level $n+\theta$ are given by linear interpolation, $U^{n+\theta}=\theta U^{n+1}+(1-\theta) U^{n}$. The charge density, $\rho^{n+\theta}$, and current density, $\mathbf{J}^{n+1 / 2}=\left(\mathbf{J}^{n+1}+\mathbf{J}^{n}\right) / 2$, are obtained from approximations to the charge continuity and momentum equations, Eqs. (2) and (3),

$$
\frac{\rho^{n+1}-\rho^{n}}{\Delta t}+\nabla \cdot \mathbf{J}^{n+\frac{1}{2}}=0
$$

and

$$
\frac{\mathbf{J}^{n+1}-\mathbf{J}^{n}}{\Delta t}=\frac{q}{m}\left[\rho^{n} \mathbf{E}^{n+\theta}+\frac{\mathbf{J}^{n+\frac{1}{2}} \times \mathbf{B}^{n}}{c}-\nabla \cdot \mathbf{P}^{n}\right] .
$$

Using the electric and magnetic field obtained by the solution of Maxwell's equations (31) in the context of the fluid moment approximation, it is possible to update explicitly the particle positions. The correction to the electric field $\mathbf{E}^{n}$, required to satisfy Eq. (11) with $\chi=0$ and $\rho^{n}$ given by particle data, is accomplished by projection

$$
\hat{\mathbf{E}}^{n}=\mathbf{E}^{n}-\nabla \phi
$$

which requires the solution of a Poisson's equation with constant coefficients for $\phi$,

$$
\nabla^{2} \phi=\nabla \cdot \mathbf{E}^{n}-4 \pi \rho^{n}
$$

where $\hat{\mathbf{E}}^{n}$ is the value of the electric field in accordance with particle data.

The numerical method employed by the implicit PIC code CELESTE3D requires $\mathbf{J}^{n+1 / 2}$ to be split as [59]

$$
\mathbf{J}^{n+1 / 2}=\hat{\mathbf{J}}^{n}+\frac{\boldsymbol{\mu}^{n}}{4 \pi \theta \Delta t} \cdot \mathbf{E}^{n+\theta},
$$

where $\hat{\mathbf{J}}^{n}$ depends on quantities that are known at time $t^{n}$ and is separated from a term which depends linearly on the electric field $\mathbf{E}^{n+\theta}$ through the tensor $\boldsymbol{\mu}^{n}$.

In particular, the case $\boldsymbol{\mu}=0$ corresponds, in the context of the implicit plasma simulations, either to a vacuum or to $\Delta t=0$. In the code CELESTE3D, $\boldsymbol{\mu}=\sum \boldsymbol{\mu}_{s}$, where the sum is over all the species of the plasma,

$$
\boldsymbol{\mu}_{s}^{n}=\frac{\theta \Delta t^{2}}{2} \omega_{\mathrm{p} s}^{2} \Pi^{n}
$$


$\omega_{\mathrm{p} s}$ is the Langmuir frequency for species $s$,

$$
\omega_{\mathrm{p} s}^{2}=\frac{4 \pi \rho_{s}^{n} q_{s}}{m_{s}}
$$

and

$$
\mathbf{\Pi}^{n}=\frac{\left[\mathbf{I}-\beta_{s} \mathbf{I} \times \mathbf{B}^{n}(\mathbf{r}) / c+\beta_{s}^{2} \mathbf{B}^{n}(\mathbf{r}) \mathbf{B}^{n}(\mathbf{r}) / c^{2}\right]}{1+\left[\beta_{s} B^{n}(\mathbf{r}) / c\right]^{2}},
$$

with I denoting the identity dyadic, $q_{s}$ and $m_{s}$ denoting the charge and the mass of particles of species $s$, and $\beta_{s}=\left(q_{s} \Delta t\right) /\left(2 m_{s}\right)$. The current density which appears in Eq. (36) is written $\hat{\mathbf{J}}^{n}=\Pi^{n} \cdot\left[\mathbf{J}^{n}-\Delta t\left(\nabla \cdot \mathbf{P}^{n}\right) / 2\right]$.

From the charge continuity equation, Eq. (32), the equation for $\mathbf{J}^{n+1 / 2}$, Eq. (36), and Maxwell's equations, Eq. (31), we derive Poisson's equation in the form that is solved in earlier versions of CELESTE3D [36],

$$
\nabla \cdot \mathbf{E}^{n+\theta}=4 \pi \hat{\rho}^{n}-\nabla \cdot\left(\boldsymbol{\mu}^{n} \cdot \mathbf{E}^{n+\theta}\right),
$$

where $\hat{\rho}$ is defined as

$$
\hat{\rho}^{n}=\rho^{n}-(\Delta t \theta) \nabla \cdot \hat{\mathbf{J}}^{n}
$$

The dispersion and stability properties of these equations are discussed in Refs. [9, 11, 59].

The solution of Eq. (40) is troublesome for two reasons. (Essentially the same equation appears in the direct implicit formulation [22, 34], so the problem is not unique to the implicit moment method.)

First, Eq. (40) is hard to solve. Various methods have been used to solve this equation. In Ref. [9], a Picard iteration is used in which the operator on the LHS is inverted using Fourier transforms, and the RHS is lagged. In Ref. [4], the electric field is decomposed using a Hodge decomposition, and a sequence of Fourier transforms is used to solve the equation. In previous versions of CELESTE3D, a matrix-free GMRES algorithm is used [48]. In Ref. [29], a matrix-free Newton-Krylov method with a multigrid preconditioner yields optimal scaling results for a simulation of an unmagnetized plasma. In Ref. [59], a direct solution in 1D is performed using Gaussian elimination. Only the last method works for arbitrary values of $\Delta t$.

Second and more importantly, the presence of $\boldsymbol{\mu}$ in Eq. (40) entangles the transverse and longitudinal parts of the electric field. The self-consistent solution of Poisson's equation, Eq. (40), and Faraday's law, Eq. (43), requires the solution of four equations for three unknowns. Introducing a scalar potential correction, as in Eq. (35), brings the number of unknowns into correspondence with the number of equations, but the presence of $\boldsymbol{\mu}$ couples the transverse and longitudinal parts of the electric field. One can show this by decomposing $\mathbf{E}$ into transverse and longitudinal parts, written $\mathbf{E}=\mathbf{E}_{1}+\mathbf{E}_{\mathrm{t}}$, where $\nabla \cdot \mathbf{E}_{\mathrm{t}}=$ 0 and $\nabla \times \mathbf{E}_{1}=0 . \mathbf{E}_{1}$ can be replaced by the gradient of the scalar potential. However, in contrast to Eq. (35), where only $\mathbf{E}_{1}$ contributes, both longitudinal and transverse fields contribute in Eq. (40) [12],

$$
\nabla \cdot \mathbf{E}_{1}^{n+\theta}=4 \pi \hat{\rho}^{n}-\nabla \cdot\left(\boldsymbol{\mu}^{n} \cdot \mathbf{E}_{1}^{n+\theta}\right)-\left(\nabla \cdot \boldsymbol{\mu}^{n}\right) \cdot \mathbf{E}_{\mathrm{t}}^{n+\theta} .
$$


As long as $\boldsymbol{\mu}$ is spatially varying, both $\mathbf{E}_{\mathrm{t}}$ and $\mathbf{E}_{1}$ appear in both Eq. (40) and Eq. (43). Therefore, it is only approximately correct to satisfy Eq. (40) by projection to correct the electric fields as described in Ref. [12].

We note that the need for solving Poisson's equation, Eq. (40), is eliminated in an approximate way in an earlier two-dimensional implicit moment scheme [42].

Substituting Ampère's law into the curl of Faraday's law, Eq. (31), yields

$$
(c \theta \Delta t)^{2} \nabla \times \nabla \times \mathbf{E}^{n+\theta}+\epsilon^{n} \cdot \mathbf{E}^{n+\theta}=\mathbf{E}^{n}+(c \theta \Delta t)\left(\nabla \times \mathbf{B}^{n}-\frac{4 \pi}{c} \hat{\mathbf{J}}^{n}\right),
$$

where $\boldsymbol{\epsilon} \equiv \mathbf{I}+\boldsymbol{\mu}$ is the implicit permittivity, a general tensor which reduces to $\mathbf{I}$ for $\Delta t \rightarrow 0$.

Using the vector identity, Eq. (18), and Poisson's equation, Eq. (40), one can write Eq. (43) in the equivalent form

$$
\begin{aligned}
& (c \theta \Delta t)^{2}\left[-\nabla^{2} \mathbf{E}^{n+\theta}-\nabla \nabla \cdot\left(\boldsymbol{\mu}^{n} \cdot \mathbf{E}^{n+\theta}\right)\right]+\boldsymbol{\epsilon}^{n} \cdot \mathbf{E}^{n+\theta} \\
& \quad=\mathbf{E}^{n}+(c \theta \Delta t)\left(\nabla \times \mathbf{B}^{n}-\frac{4 \pi}{c} \hat{\mathbf{J}}^{n}\right)-(c \theta \Delta t)^{2} \nabla 4 \pi \hat{\rho}^{n} .
\end{aligned}
$$

Both Eqs. (44) and (43) are used in implicit plasma simulation. Kamimura et al. solve Eq. (43) [27]. On the other hand, Hewett and Langdon find that a formulation consistent with Eq. (44) provides better performance with an alternating direction implicit solver [22]. Our analysis provides two additional reasons for preferring Eq. (44) to Eq. (43).

The analysis of the evolution of the divergence constraint provides the first reason. The function $\chi$ is discretized with values $\chi^{n}(\mathbf{r})$ and Fourier component amplitude $\chi_{\mathbf{k}}^{n}$. Applying the divergence operator to Eq. (43) gives

$$
\chi_{\mathbf{k}}^{n+1}=\chi_{\mathbf{k}}^{n}
$$

so that an initial perturbation of the Gauss law neither grows nor decays but remains constant in time. On the other side, the analysis of the evolution of the divergence conditions for Eq. (44) leads to

$$
\chi_{\mathbf{k}}^{n+1}=\chi_{\mathbf{k}}^{n}\left[1-\frac{1}{\theta} \frac{(c \Delta t k)^{2}}{(c \Delta t k)^{2}+1}\right]
$$

and $\left|\chi_{\mathbf{k}}^{n+1}\right|<\left|\chi_{\mathbf{k}}^{n}\right|$, for every $\mathbf{k}$ and for $\theta \geq 1 / 2$. This means that the initial perturbations of the Gauss law are damped, as is shown in Fig. 1.

The second reason is numerical. Equation (44) leads to a system of linear equations which is better conditioned than Eq. (43). In fact, using the vector identity, Eq. (18), the LHS of Eqs. (43) and (44) can be compared: Their only difference lies in the term $\nabla \nabla \cdot \mathbf{E}^{n+\theta}$, which is replaced by $-\nabla \nabla \cdot\left(\boldsymbol{\mu} \cdot \mathbf{E}^{n+\theta}\right)$ in Eq. (44). In Eq. (43), the term $\nabla \nabla \cdot \mathbf{E}^{n+\theta}$ decreases the absolute value of the diagonal elements, while the opposite effect is shown in Eq. (44). Thus, Eq. (44) is associated with a matrix whose condition number is smaller than that for Eq. (43) and its numerical solution is easier to obtain.

The boundary conditions for Eqs. (43) and (44) can be obtained directly from system (14). Regarding the electric wall, it is possible to write

$$
\left\{\begin{array}{l}
\mathbf{n} \times \mathbf{E}^{n+\theta}=0 \\
\nabla \cdot\left(\boldsymbol{\epsilon}^{n} \cdot \mathbf{E}^{n+\theta}\right)=4 \pi \hat{\rho}^{n}
\end{array}\right.
$$




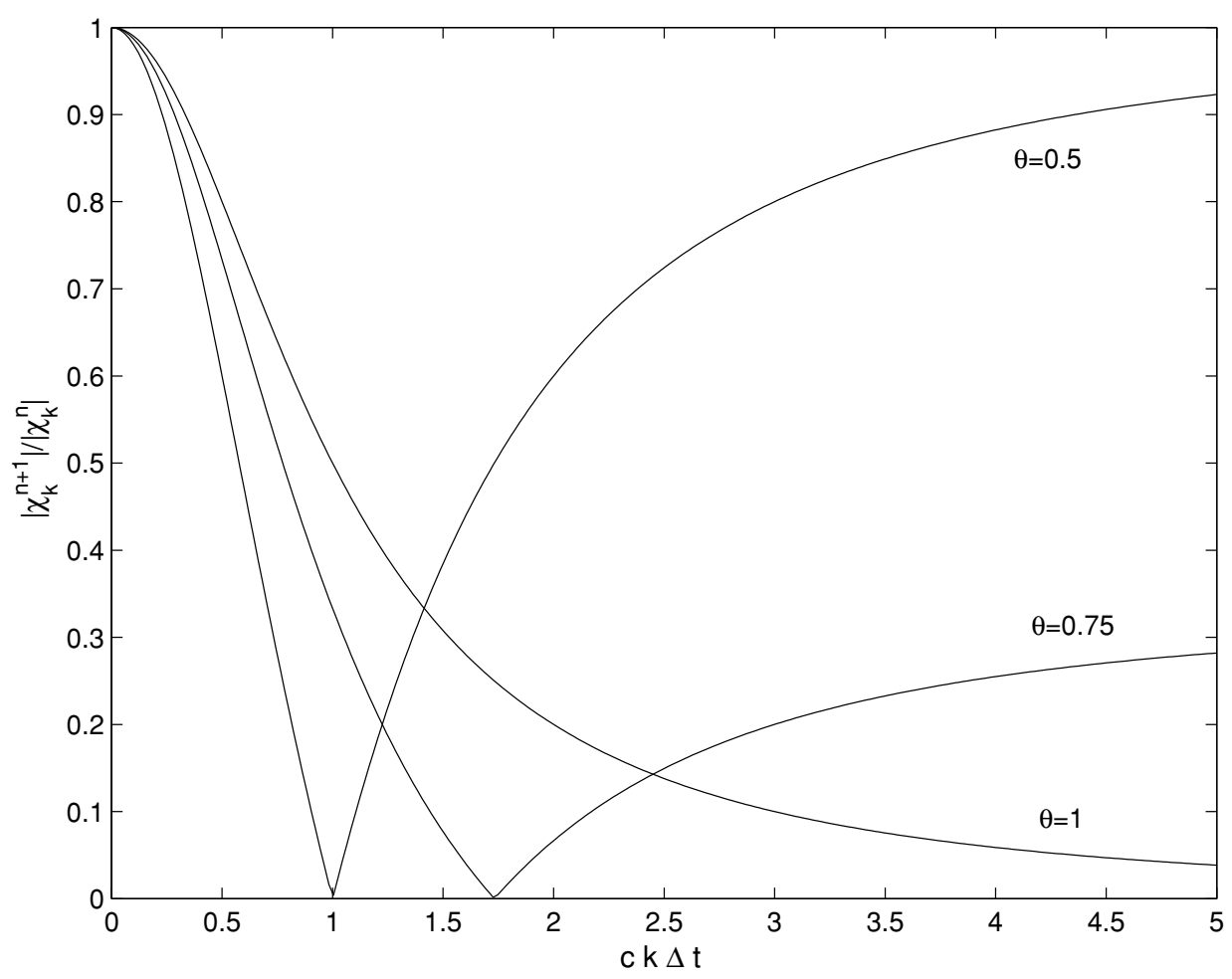

FIG. 1. Ratio of the divergence errors $\left(\chi_{k}\right)$ between two consecutive time steps, as shown by Eq. (46), when system (44) is considered, for different values of $\theta$ and as a function of $c k \Delta t$.

where we note that the implicit Poisson's equation need be solved only on the boundary $\partial \Omega_{1}$, not in the whole domain as was necessary previously.

On the magnetic wall, the boundary conditions can be written readily as

$$
\left\{\begin{array}{l}
\mathbf{n} \cdot \mathbf{E}^{n+\theta}=0 \\
\mathbf{n} \times\left(\nabla \times \mathbf{E}^{n+\theta}\right)=0 .
\end{array}\right.
$$

We note that Eq. (44) has no spurious solutions satisfying the equation

$$
(c \theta \Delta t)^{2}\left[-\nabla^{2} \delta-\nabla \nabla \cdot\left(\mu^{n} \cdot \delta\right)\right]+\epsilon^{n} \cdot \delta=0,
$$

which is derived from Eq. (44) by setting the RHS to zero. We show that the only solution to this equation is $\delta=0$.

In general, the solution $\delta$ is such that $\nabla \cdot\left(\epsilon^{n} \cdot \delta\right)=0$ [the boundary conditions (47) and (48) with the resulting divergence constraint are satisfied], and so Eq. (49) can be simplified as

$$
(c \theta \Delta t)^{2}[\nabla \times \nabla \times \delta]+\epsilon^{n} \cdot \delta=0 .
$$

Consider the integral $I$, defined as

$$
I=\int_{\Omega} \delta \cdot\left((c \theta \Delta t)^{2}[\nabla \times \nabla \times \delta]+\boldsymbol{\epsilon} \cdot \boldsymbol{\delta}\right) d \mathbf{r}=0,
$$



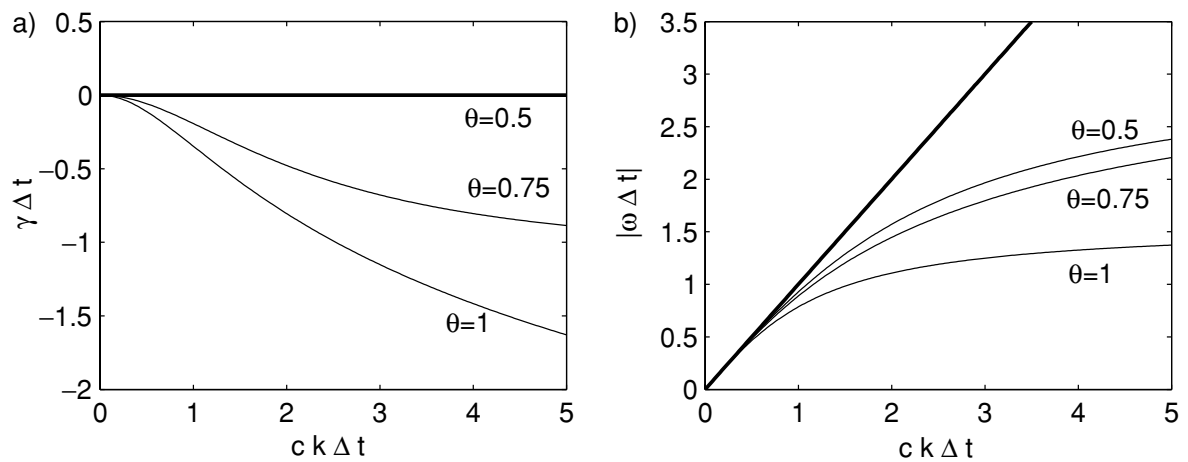

FIG. 2. Numerical dispersion relation of the electromagnetic waves given by the discretized Eqs. (43) and (44), for different values of $\theta$ and as a function of $c k \Delta t$. The thick solid lines represent the analytical dispersion relation.

which is zero because Eq. (50) is satisfied. Using a standard vector identity, the triple vector product in Eq. (51) is replaced by

$$
(\nabla \times \delta) \cdot(\nabla \times \delta)=\delta \cdot(\nabla \times(\nabla \times \delta))+\nabla \cdot(\delta \times \nabla \times \delta) .
$$

Since the boundary conditions require either $\mathbf{n} \times \delta=0$ on $\partial \Omega_{1}$ or $\mathbf{n} \times(\nabla \times \delta)=0$ on $\partial \Omega_{2}$, the contribution of the second term in Eq. (52) to the integral in Eq. (51) is zero,

$$
\int_{\Omega} \nabla \cdot(\boldsymbol{\delta} \times(\nabla \times \delta)) d \mathbf{r}=\int_{\partial \Omega} \mathbf{n} \cdot(\boldsymbol{\delta} \times \nabla \times \delta) d S=0 .
$$

Using the definition of $\epsilon$, Eq. (37), and the fact that $\delta \cdot \Pi_{s} \cdot \delta=\delta \cdot \delta$, Eq. (51) is replaced by

$$
I=\int_{\Omega}\left[(\nabla \times \delta) \cdot(\nabla \times \delta)+\delta \cdot \delta\left(1+\sum_{s} \frac{\theta \Delta t^{2}}{2} \omega_{\mathrm{p} s}^{2}\right)\right] d \mathbf{r}=0
$$

Since both terms in the integrand are positive and $I=0$, it follows that $\delta=0$ is the only solution of Eq. (49) in the domain $\Omega$.

With $\mathbf{E}^{n+\theta}$ computed, the magnetic field is evaluated from Faraday's law, Eq. (31), as

$$
\frac{\left(\mathbf{B}^{n+1}-\mathbf{B}^{n}\right)}{c \Delta t}=-\nabla \times \mathbf{E}^{n+\theta}
$$

The dispersion relation of electromagnetic waves given by the time-discretized system is plotted in Fig. 2 [11]. We note that the dispersion would be considered unacceptable in computational electromagnetics, even with $\theta=1 / 2$. However, in plasma simulations, which are driven by noisy particle data, the adjustable parameter $\theta$ provides useful filtering and damping of poorly resolved, high-frequency modes. The role of $\theta$ in energy conservation is discussed in [9]. Filtering for higher order time differencing is discussed in [15, 47].

\subsection{Spatial Discretization}

Maxwell's equations are discretized in space using a uniform grid with spacing $\Delta x, \Delta y$, and $\Delta z$ in the $x, y$, and $z$ directions. The vertices of the mesh which resolve $\Omega=\left[0, L_{x}\right] \times$ 
$\left[0, L_{y}\right] \times\left[0, L_{z}\right]$ are located at $\mathbf{r}_{i j k}=(i \Delta x, j \Delta y, k \Delta z)$, where $i \in\left[0, N_{x}\right], j \in\left[0, N_{y}\right], k \in$ $\left[0, N_{z}\right]$. The electric field $\mathbf{E}^{n}$, the current density $\mathbf{J}^{n}$, and the permittivity $\boldsymbol{\epsilon}^{n}$ are evaluated at the vertices of the mesh. The magnetic field $\mathbf{B}^{n}$ and charge density $\rho^{n}$ are evaluated at the centers of cells, labeled $\mathbf{r}_{i+1 / 2, j+1 / 2, k+1 / 2}$.

The differencing for CELESTE3D, which is described in Ref. [54], can be used for a nonorthogonal, logically rectangular grid. However, the numerical algorithm for solving Maxwell's equations requires that the discrete analogues of the vector identities $\nabla \times \nabla f=$ 0 and $\nabla \cdot \nabla \times \mathbf{v}=0$ be satisfied exactly. This requirement is met by the CELESTE3D differencing only on a uniform grid.

\subsubsection{The Difference Equations}

Derivatives are approximated by a simple box scheme. Where $\psi_{i j k}$ is given at vertices, $\partial \psi / \partial x$ at cell centers is given by

$$
\left.\frac{\partial \psi}{\partial x}\right|_{i+1 / 2, j+1 / 2, k+1 / 2}=\frac{\psi_{i+1, j+1 / 2, k+1 / 2}-\psi_{i, j+1 / 2, k+1 / 2}}{\Delta x},
$$

and similarly for $\partial \psi / \partial y$ and $\partial \psi / \partial z$. The face-centered value of $\psi$ is computed by averaging the neighboring vertex values,

$$
\psi_{i, j+1 / 2, k+1 / 2}=\frac{1}{4}\left(\psi_{i j k}+\psi_{i, j+1, k}+\psi_{i, j+1, k+1}+\psi_{i, j, k+1}\right) .
$$

Derivatives of variables stored at the centers of cells are approximated similarly. For example, given $\lambda_{i+1 / 2, j+1 / 2, k+1 / 2}$, the derivative $(\partial \lambda / \partial x)_{i j k}$ is approximated by

$$
\left.\frac{\partial \lambda}{\partial x}\right|_{i j k}=\frac{\lambda_{i+1 / 2, j, k}-\lambda_{i-1 / 2, j, k}}{\Delta x},
$$

where

$\lambda_{i+1 / 2, j, k}=\frac{1}{4}\left(\lambda_{i+1 / 2, j+1 / 2, k+1 / 2}+\lambda_{i+1 / 2, j+1 / 2, k-1 / 2} \lambda_{i+1 / 2, j-1 / 2, k-1 / 2}+\lambda_{i+1 / 2, j-1 / 2, k+1 / 2}\right)$.

With these expressions, the field equations, Eqs. (43) and (44), are evaluated in a straightforward manner. In particular, the discrete analog of the Laplacian operator is obtained by combining the divergence and gradient operators. On a two-dimensional grid, the Laplacian has a five-point stencil; the central point is coupled to the four corners. On a threedimensional grid, the Laplacian has a nine-point stencil: the central point and the eight corners of the cube around the central point.

\subsubsection{Boundary Conditions}

The boundary conditions expressed in Eqs. (47) and (48) are differenced in a similar fashion.

For simplicity of presentation, we describe both conductor and constant magnetic flux surface conditions for the same boundary $z=L_{z}$. The application to other boundaries is straightforward. 
The perfect conductor boundary condition given by Eq. (47), $\mathbf{n} \times \mathbf{E}=0$, becomes $E_{x}\left(x, y, L_{z}\right)=E_{y}\left(x, y, L_{z}\right)=0$, which means $E_{x, i, j, N_{z}}=E_{y, i, j, N_{z}}=0$. Regarding the divergence constraint, we note that the charge density $\hat{\rho}$ is zero inside the conductor, and an integral over a volume enclosing a portion of the boundary will contain contributions from the interior of $\Omega$ only. At $k=N_{z}$, one obtains the following expression:

$$
\begin{aligned}
\left(D_{z, i+1 / 2, j+1 / 2, N_{z}}-D_{z, i+1 / 2, j+1 / 2, N_{z}-1}\right) \Delta x \Delta y \\
+\left(D_{x, i+1, j+1 / 2, N_{z}-1 / 2}-D_{x, i, j+1 / 2, N_{z}-1 / 2}\right) \Delta y \Delta z \\
+\left(D_{y, i+1 / 2, j+1, N_{z}-1 / 2}-D_{y, i+1 / 2, j, N_{z}-1 / 2}\right) \Delta z \Delta x \\
=4 \pi \hat{\rho}_{i+1 / 2, j+1 / 2, N_{z}-1 / 2} \Delta x \Delta y \Delta z .
\end{aligned}
$$

Recall that the two tangential components of the electric field at the boundary are equal to zero, and the only unknown quantities in Eq. (60) are the normal components of the electric field at the vertices with $k=N_{z}$, which lie on the boundary. In general, one must evaluate the normal components of the electric field by solving the coupled linear system given by Eqs. (60) for all the boundary cells.

However, in particular cases, because to the properties of the tensor $\boldsymbol{\mu}$, the system made up by the set of Eqs. (60) can be decoupled. This is the case for the GEM challenge, where a two-dimensional system is considered with a negligible out-of-plane magnetic field on the conducting boundaries. In this case, $\mathbf{B B} \cdot \mathbf{E}=0$ and the in-plane component of $(\mathbf{I} \times \mathbf{B}) \cdot \mathbf{E}$ parallel to the boundary vanishes so that the in-plane $\mathbf{E}$ and $\mathbf{D}$ are parallel. It follows that the vector $\mathbf{D}$ is normal to the boundary and Eq. (60) becomes a simple Neuman boundary condition for $D_{z}$ and, as a consequence, for $E_{z}$.

Moreover, at the cost of an additional error, the divergence boundary constraint can be expressed using a first-order discretization scheme, with which it is possible to decouple the system of Eqs. (60). The error this introduces is discussed in the text section while analyzing some test cases.

The magnetic mirror boundary condition, Eq. (48), can be applied in a similar fashion. If the boundary $z=L_{z}$ represents a magnetic mirror, $E_{z, i j N_{z}}=0$ and the normal derivatives of the tangential components of $\mathbf{E}$ are equal to zero. Thus, the boundary conditions can be summarized as

$$
\left\{\begin{array}{l}
E_{x, i, j, N_{z}}=E_{x, i, j, N_{z}-1} \\
E_{y, i, j, N_{z}}=E_{y, i, j, N_{z}-1} \\
E_{z, i, j, N_{z}}=0,
\end{array}\right.
$$

which, we remark, are first-order boundary conditions.

\section{NUMERICAL RESULTS}

First, a numerical code has been developed to test the Maxwell solver without plasma in two dimensions. Some simple test cases have been considered to study in detail the properties of the numerical algorithm. The test cases are two dimensional, with $\boldsymbol{\mu}=0$; they involve transverse electric (TE) waves. In this particular case, analytical solutions of Maxwell's equations are available. This test case shows that Jiang et al.'s boundary conditions, which were implemented and tested with a finite element method, also work 
with finite differences. In particular, we measure the accuracy with which Gauss's law is satisfied and how the error is affected by the accuracy of the boundary conditions.

Second, the Maxwell solver developed here is applied to the implicit PIC code CELESTE3D, which requires the solution of the Maxwell equation in media with anisotropic and inhomogeneous $\boldsymbol{\mu}$. The numerical simulations shown concern the GEM magnetic reconnection challenge [7].

\subsection{Numerical Results for Simple Test Cases}

A two-dimensional charge-free system is considered, with $\Omega=\left[0, L_{x}\right] \times\left[0, L_{y}\right]$, denoting a generic point in the domain $\Gamma$ with $(x, y, t)$. The TE waves are considered, so that $\mathbf{E}=\left(E_{x}, E_{y}, 0\right)$ and $\mathbf{B}=\left(0,0, B_{z}\right)$. The boundary conditions are set, on the edges $x=0$ and $y=L_{y}$, as perfect conductors; the edges $y=0$ and $x=L_{x}$ are magnetic flux surfaces. The natural boundary conditions for this system can be expressed as $E_{y}=0$ for $x=0$, $E_{x}=0$ for $y=L_{y}, E_{y}=0$ for $y=0$, and $E_{x}=0$ for $x=L_{x}$. The additional boundary conditions set for the second-order system are $\partial E_{x} / \partial x=0$ for $x=0, \partial E_{y} / \partial y=0$ for $y=L_{y}, \partial E_{x} / \partial y=0$ for $y=0$, and $\partial E_{y} / \partial x=0$ for $x=L_{x}$.

It is possible to solve analytically Maxwell's equation inside this physical system. The analytical solution considered can be expressed as

$$
\left\{\begin{array}{l}
E_{x}(x, y, t)=E_{0} k_{y} \cos \left(k_{x} x\right) \cos \left(k_{y} y\right) \sin (k c t) / k_{x} \\
E_{y}(x, y, t)=E_{0} \sin \left(k_{x} x\right) \sin \left(k_{y} y\right) \sin (k c t) \\
B_{z}(x, y, t)=E_{0} k \cos \left(k_{x} x\right) \sin \left(k_{y} y\right) \cos (k c t) / k_{x}
\end{array}\right.
$$

where

$$
k_{x}=\frac{\pi}{2 L_{x}}, \quad k_{y}=\frac{\pi}{2 L_{y}}
$$

Figure 3 compares the analytical and numerical solutions of Maxwell's equations for electromagnetic waves (62) for different values of the parameter $\theta$. The numerical solutions show no damping for the case $\theta=1 / 2$. With other values $(\theta=3 / 4$ and $\theta=1)$, the electromagnetic waves are damped.

For the same physical system, the error in the solution of Poisson's equation for the electric field is displayed in Figs. 4 and 5. In particular, to understand the effect of the

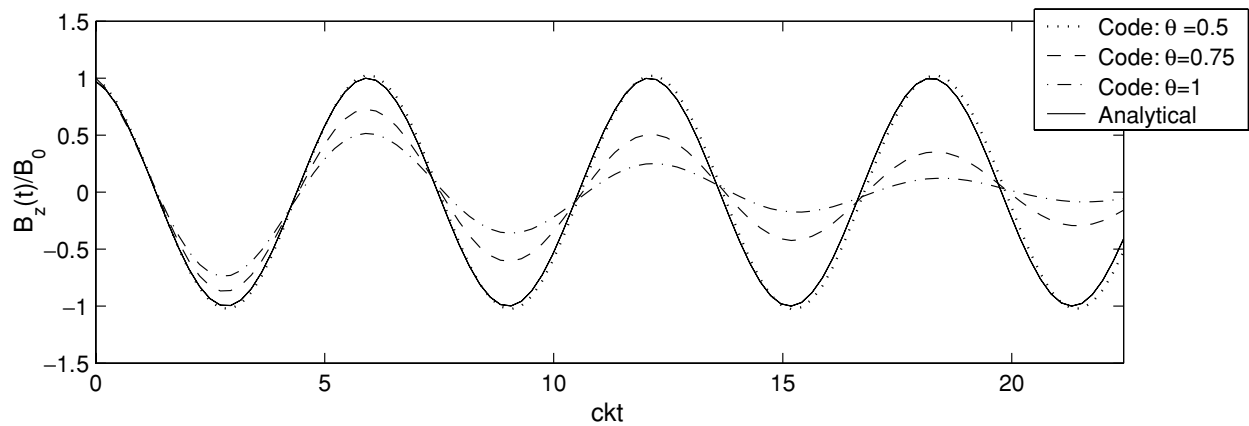

FIG. 3. Evolution of the magnetic field $B_{z}$, in a generic point of the spatial domain, for different values of the parameter $\theta$. The case represented is that of Eq. (62). It is $L_{x}=L_{y}, c k \Delta t=0.25$, and $k \Delta x=k \Delta y=0.045$. 
a)

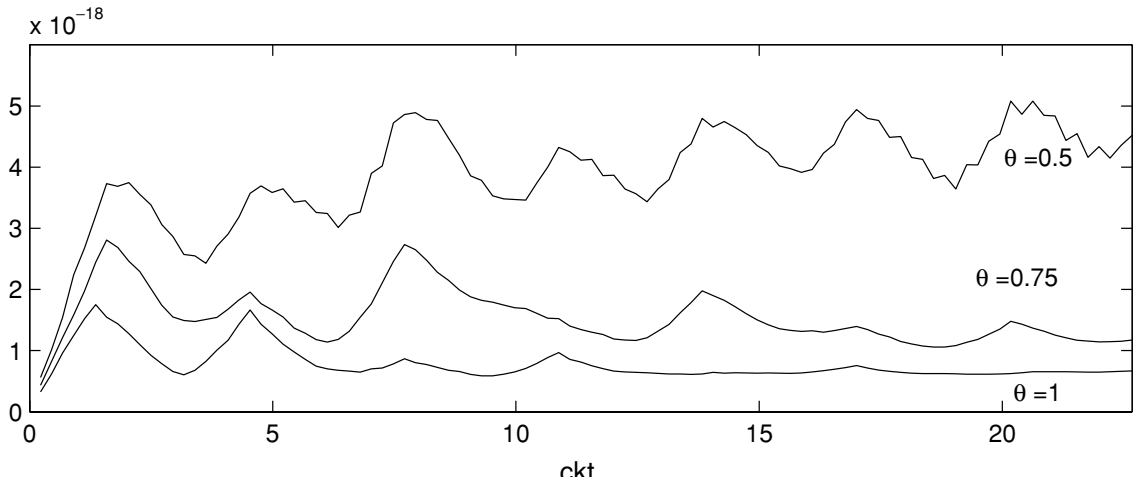

b)

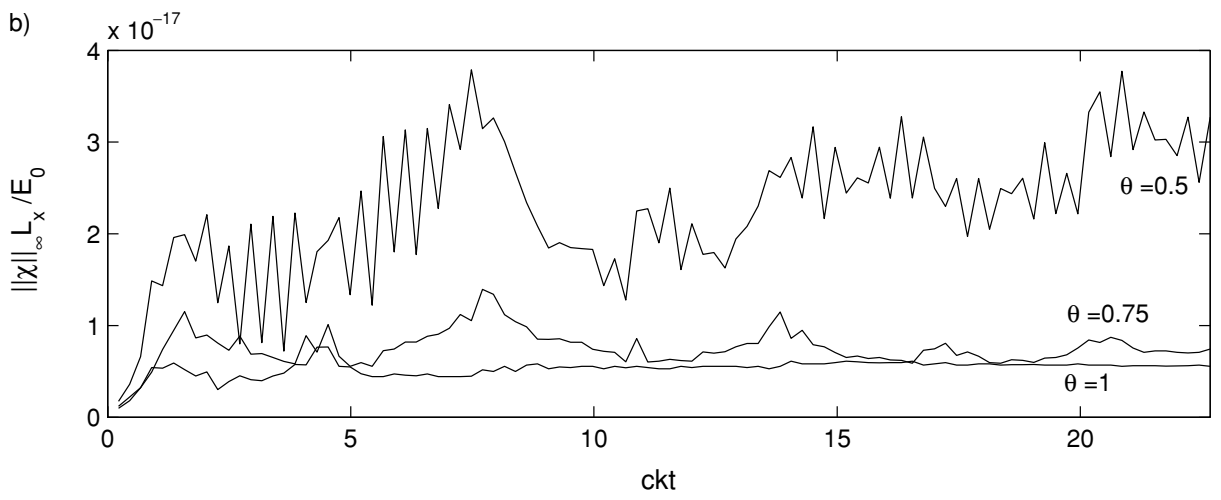

FIG. 4. (a) Evolution of the $\mathrm{L}_{2}$-norm and (b) evolution of the $\mathrm{L}_{\infty}$-norm of the function $\chi$, for different values of the parameter $\theta$, for the same system represented in Fig. 3.

accuracy of the boundary conditions, two different formulations of the boundary conditions are implemented. (We remark that the boundary conditions can be simplified with respect to the general case, as $\boldsymbol{\mu}=0$ and $\rho=0$.) The first formulation satisfies the boundary conditions to first-order accuracy for physical boundaries that pass through the vertices of the grid. For the perfect conductor at $x=0$, the boundary conditions $E_{y, 0, j}=0$ and $E_{x, 0, j}=E_{x, 1, j}$ are applied. For the magnetic flux surface at $y=0$, we apply the boundary conditions $E_{y, i, 0}=0$ and $E_{x, i, 0}=E_{x, i, 1}$. Similar conditions are applied to the other two edges. The second formulation satisfies the boundary conditions to second-order accuracy for physical boundaries that pass through the cell centers. At $x=0$, the boundary conditions $E_{y, 0, j}=-E_{y, 1, j}$ and $E_{x, 0, j}=E_{x, 1, j}$ are applied; while for $y=0$, we apply the boundary conditions $E_{y, i, 0}=-E_{y, i, 1}$ and $E_{x, i, 0}=E_{x, i, 1}$. The boundary conditions for the other two edges are applied similarly.

In Fig. 4 the numerical errors related to the divergence constraints are shown using the $\mathrm{L}_{2}$-norm, defined as

$$
\|\chi\|_{2}=\frac{1}{N} \sqrt{\sum_{c}\left(\chi_{c}\right)^{2}},
$$

where the sum is extended to all the cell centers and $N$ denotes the number of cell centers in the system. Also the $\mathrm{L}_{\infty}$-norm, defined as

$$
\|\chi\|_{\infty}=\max _{c}\left(\chi_{c}\right),
$$



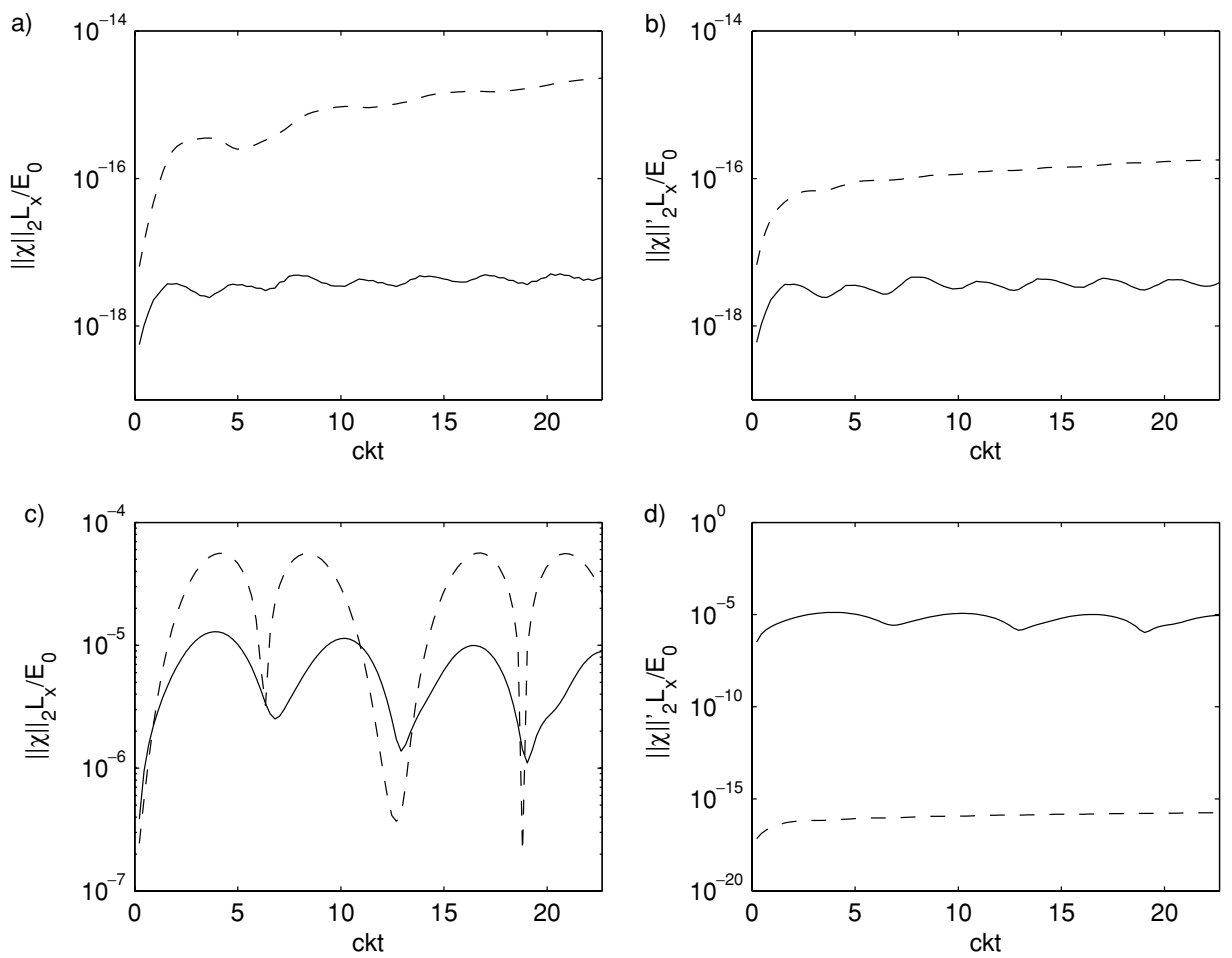

FIG. 5. (a), (c) Evolution of the $\mathrm{L}_{2}$-norm and (b), (d) evolution of the $\mathrm{L}_{2}^{\prime}$-norm of the function $\chi$, when system (43) is implemented (dashed lines) and when system (44) is considered (solid lines). In the upper figures [(a) and (b)], the boundaries are of second order, in the lower figures [(c) and (d)] the boundaries are of first order. The physical system represented in Fig. 3 is considered.

has been employed. In this case, the code implements Eq. (44) and the physical boundaries of the system are the second-order ones. Figure 4 shows that the numerical errors related to the divergence constraint are almost constant in time and always of the order of the truncation error. The error is smaller in the case $\theta=1$ : This is due both to the fact that, in this case, the electric field is damped and to the growth rates of the error on the divergence constraint being different depending on $\theta$, as stated by Eq. (46).

Figure 5 represents the error on the divergence constraint to compare the cases when Eq. (43) or Eq. (44) is implemented, and when the boundaries are of first and second order. In Fig. 5, the $\mathrm{L}_{2}^{\prime}$-norm is employed, which is defined as

$$
\|\chi\|_{2}^{\prime}=\frac{1}{N^{\prime}} \sqrt{\sum_{c^{\prime}}\left(\chi_{c^{\prime}}\right)^{2}},
$$

where $c^{\prime}$ denotes the inner cell centers (the cells not located on the boundary) and $N^{\prime}$ is their number.

The plots of the $\mathrm{L}_{2}$-norm show that the divergence constraint is better respected when Eq. (44) is implemented. This fact agrees with the theoretical discussion developed in Eqs. (45) and (46). In particular, the $\mathrm{L}_{2}$-norm is smaller if the boundary conditions are of second order. The plots of the $\mathrm{L}_{2}^{\prime}$-norm show that, if system (43) is implemented, the divergence constraint of each cell is decoupled from the others, as follows from Eq. (45); 
in particular, the inner cells are decoupled from the boundary cells. This can be seen more clearly in the case of first-order boundary conditions. In this case, in fact, the error on the divergence constraint on the boundary is significant, being of order $10^{-6} E_{0} / L_{x}$ while, inside the domain, the divergence error is of order $10^{-17} E_{0} / L_{x}$. If Eq. (43) is implemented, the errors do not penetrate inside the domain: The $\mathrm{L}_{2}^{\prime}$-norm has the same order of magnitude which characterizes the interior domain while the $\mathrm{L}_{2}$-norm, which takes into account the boundary cells, has an order of magnitude comparable to the divergence error of the boundary cells. By contrast, if Eq. (44) is implemented, the $\mathrm{L}_{2}$-norm and the $\mathrm{L}_{2}^{\prime}$-norm are comparable.

\subsection{Testing CELESTE3D against the GEM Challenge}

The code CELESTE3D with the new Maxwell solver is tested by performing a simulation whose parameters match those of the GEM magnetic reconnection challenge (see Ref. [7] and references therein).

The GEM challenge project seeks to understand the physics that controls the rate of magnetic reconnection in the Earth's magnetotail. Through magnetic reconnection, magnetic field lines of opposite polarity reconfigure to a lower energy state, with the release of magnetic energy as heat, directed plasma flow, and the penetration of the solar wind into the magnetosphere. Magnetic reconnection in the magnetotail has been observed by the Wind spacecraft in a serendipitous encounter, which has revealed the key processes predicted by the models [44].

Within the GEM challenge, the same standard two-dimensional Harris sheet configuration, with a specified set of initial conditions, has been studied by employing a variety of codes, ranging from resistive MHD codes to fully kinetic particle codes (PIC codes). The initial parameters used and the results of the simulations are summarized in Ref. [7]. In particular, the computational domain is chosen to be rectangular $\left(-L_{x} / 2 \leq x \leq L_{x} / 2\right.$, $\left.-L_{z} / 2 \leq z \leq L_{z} / 2\right)$ with periodic boundary conditions in the $x$ direction and conducting boundaries at $z= \pm L_{z} / 2$.

Using CELESTE3D, the GEM challenge has been performed with a $N_{x} \times N_{z}=64 \times$ 64 grid, a total of $2 \times 10^{5}$ computational particles and a time step $\omega_{\mathrm{pi}} \Delta t=0.3$. For comparison, the explicit PIC simulation presented in Ref. [45] employs a $N_{x} \times N_{z}=512 \times 256$ grid, $9.12 \times 10^{6}$ computational particles, and a time step $\omega_{\mathrm{pe}} \Delta t=0.15$ (10 times smaller than ours).

The main result of the GEM challenge is that all models that include the Hall effect in the generalized Ohm's law produce similar rates of reconnection. When the Hall effect is eliminated from the simulations (resistive MHD model), the reconnection rate is drastically reduced [8]. In particular, the results of different simulations [21, 31, 45, 51] show that the difference in the ion and electron dynamics gives rise to in-plane (Hall) currents that produce an out-of-plane magnetic field with quadrupolar structure which has a key role in the magnetic reconnection.

The quadrupolar structure of the out-of-plane magnetic field is reproduced by the simulation performed with CELESTE3D, as shown in Fig. 6. (In Figs. 6-9, the distances are normalized to the ion collisionless skin depth, $c / \omega_{\mathrm{pi}}$, the magnetic field to $B_{0}$, the velocity to the Alfvén speed, $v_{\mathrm{A}}=B_{0} / \sqrt{4 \pi \rho}$, and the reconnected flux to $\omega_{\mathrm{pi}} /\left(B_{0} c\right)$.)

The structure and the peak value of the magnetic field are in quantitative agreement with the kinetic simulation shown in Plate 1 of Ref. [45]. In Fig. 7, the evolution of the 


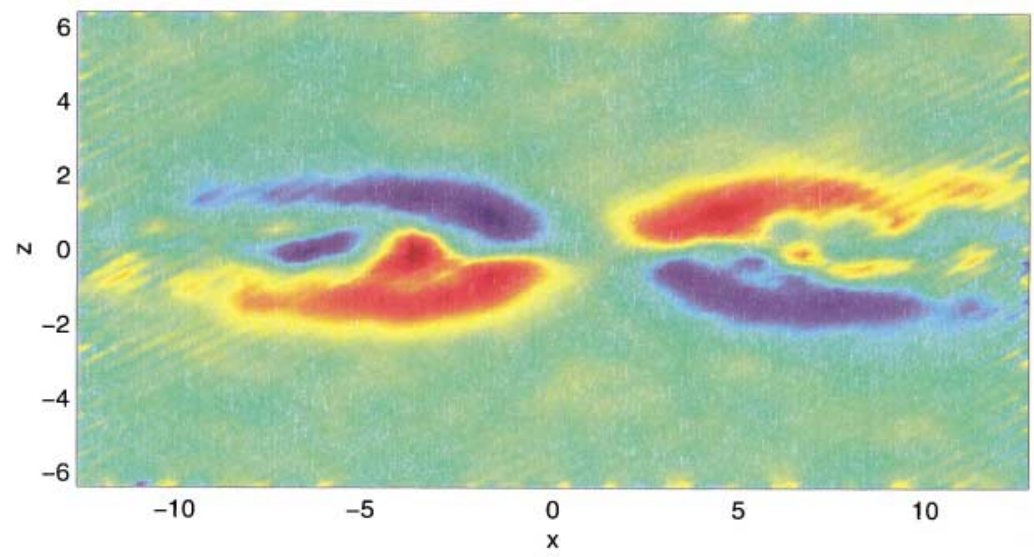

FIG. 6. Contours of the out-of-plane $B_{y}$ field at the time $\omega_{c i} t=15$ for the simulation whose parameters match those ones of the GEM challenge [7]. This figure is in agreement with Plate 1 in Ref. [45].

$t_{c}=0$

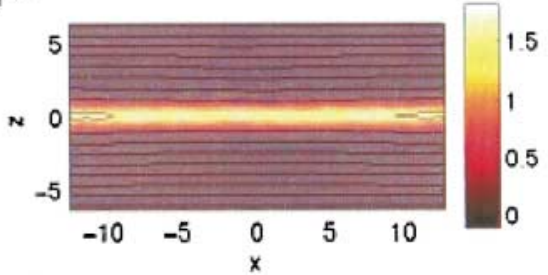

$\operatorname{tos}_{d}=16$

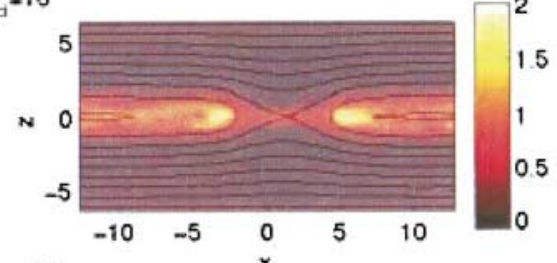

tw -32

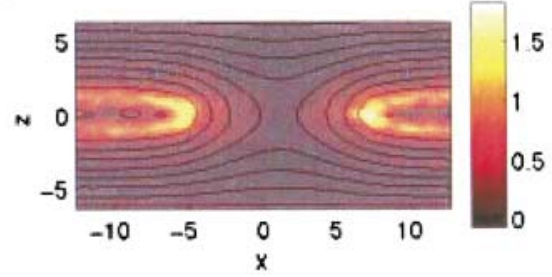

$\operatorname{ton}_{1}=8$

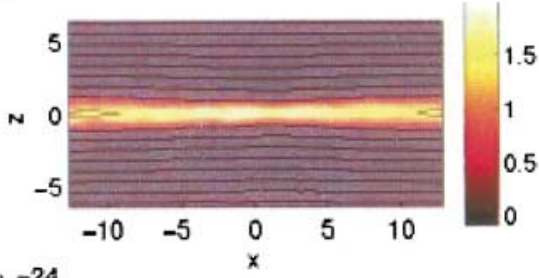

$t_{0}=24$

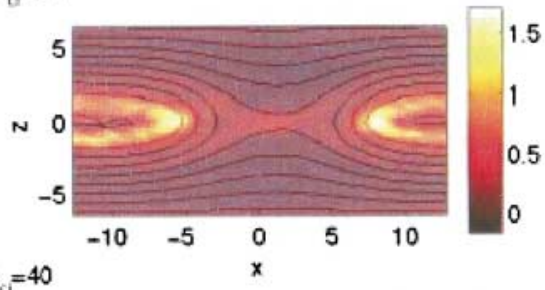

$t_{i \rightarrow}=40$

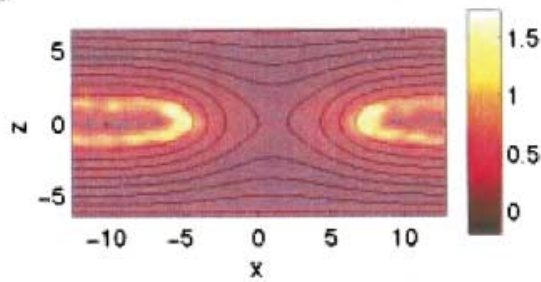

FIG. 7. Evolution of the magnetic field and evolution of the out-of-plane current density (color coded) for the simulation whose parameters match those of the GEM challenge [7]. This figure is in agreement with Plate 1 in Ref. [21]. 


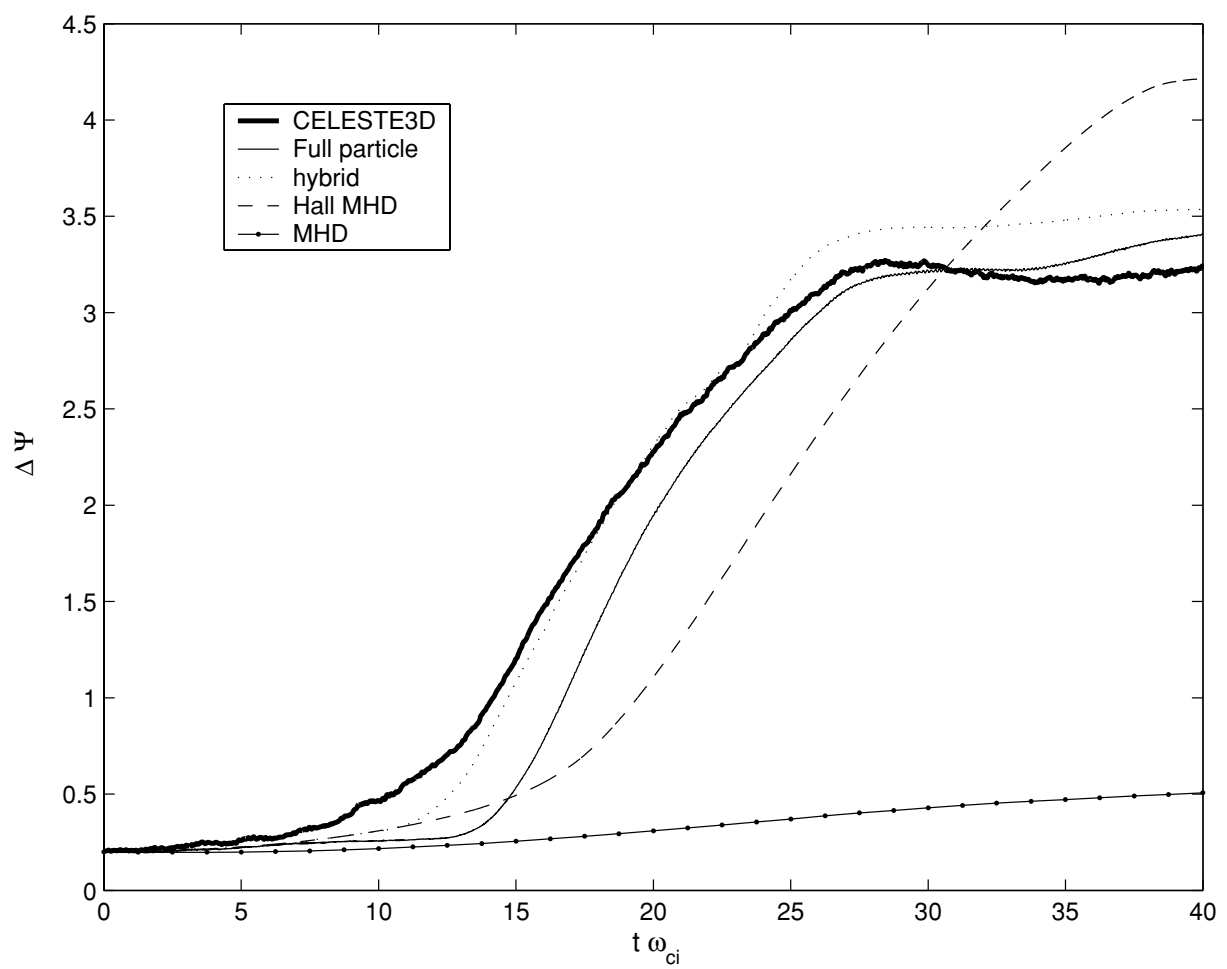

FIG. 8. Reconnected flux, $\Delta \Psi$, as a function of time, for the GEM magnetic reconnection challenge [7]. The reconnection rate reported by CELESTE3D is compared with the results (provided by J. F. Drake and M. A. Shay) of the different codes which have performed the GEM challenge (see Fig. 1 of Ref. [7]).

magnetic field lines is shown with the out-of-plane current density: The reconnection starts rapidly after the current has rearranged itself in the dissipation region. The evolution of the field lines and current density is in agreement with those shown in Plate 1 of Ref. [21]. The reconnection rate computed by CELESTE3D is plotted in Fig. 8 and compared with the results of the simulation codes used for the GEM challenge, Fig. 1 of Ref. [7], which have been provided by J. F. Drake and M. A. Shay. The results of CELESTE3D are in remarkable agreement with all the previous simulations performed by models that include the Hall effect.

Even if the reconnection rates given by all the models that include the Hall effect are similar, there are other physical quantities whose behavior is different in the fluid and the kinetic simulations. In particular, the $x$ component of the ion and electron velocities obtained by the kinetic simulations are smaller than those observed with the HallMHD model by a factor 2.5, in agreement with previous kinetic simulation results [21]. These physical quantities and the ion density, as given by CELESTE3D, are displayed in Fig. 9. The results are compared with the simulation data provided by P. L. Pritchett from Figs. 4 and 6 of Ref. [45]. The agreement between the CELESTE3D results and Pritchett's explicit simulations is remarkable. We observe that the higher fluctuations in the CELESTE3D results are a consequence of the smaller number of particles used in the implicit simulation. 

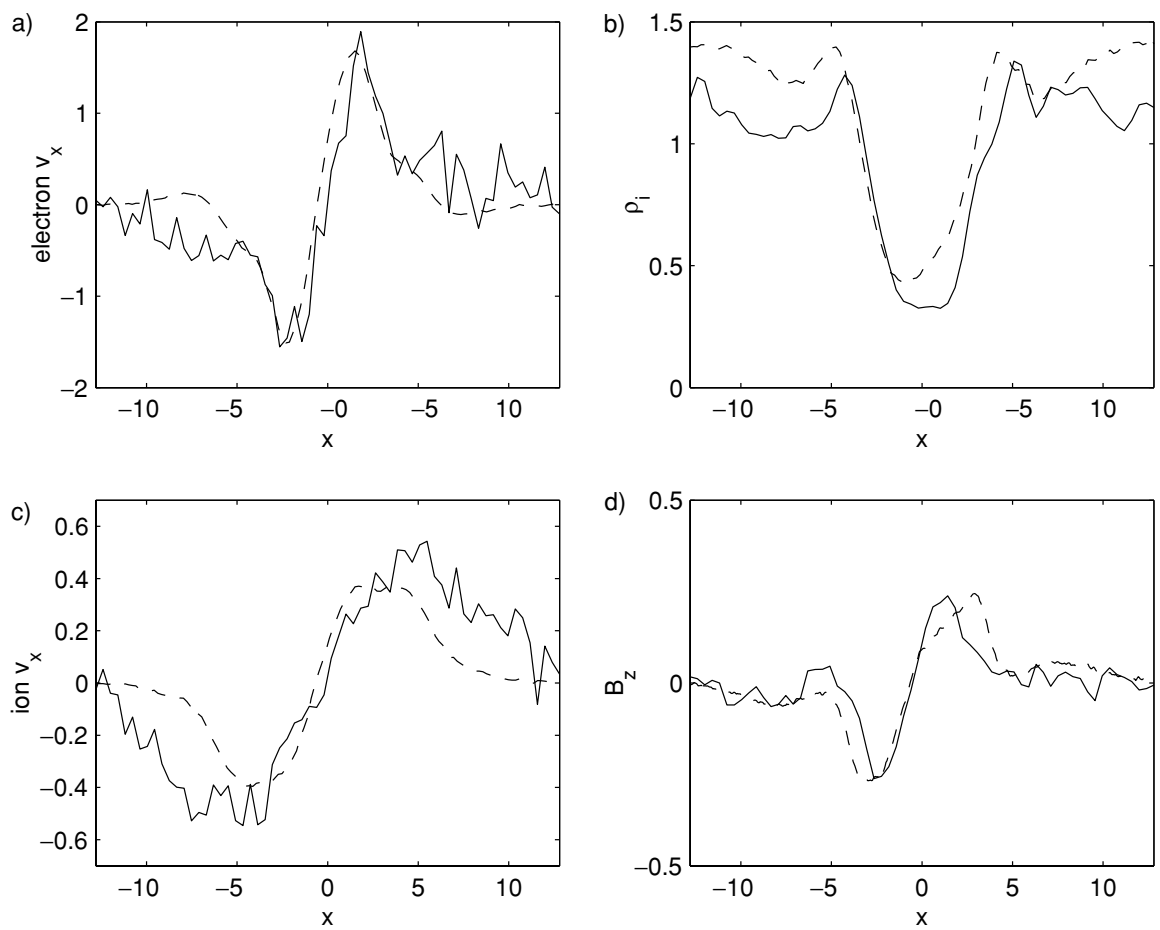

FIG. 9. Comparison between CELESTE3D (solid line) and the explicit kinetic simulation data provided by P. L. Pritchett (see Ref. [45], Figs. 4 and 6) (dashed line) of (a) the $x$ component of the electron velocity, (b) ion density, (c) $x$ component of the ion velocity, and (d) $z$ component of the magnetic field, as a function of $x$ and at $z=0$, when $\Delta \Psi=1$ [(a)-(c) averaged between $t \omega_{c i}=15$ and $t \omega_{c i}=15.6$ and (d) at $t \omega_{c i}=15$ for Pritchett's simulation] within the GEM magnetic reconnection challenge. The results reported in this figure can also be compared with Fig. 9 of Ref. [21].

\section{CONCLUSION}

Within the implicit-moment method for plasma simulations, we have presented a Maxwell solver that eliminates the need to solve an implicit Poisson's equation (40) in the volume through a careful formulation of the boundary conditions following Jiang et al. [32]. (A standard projection, Eq. (35), corrects for inconsistencies between the particle and moment data each cycle.) Because implicit formulations of Eq. (40) couple transverse and longitudinal parts of the electric field, normal projection methods are only approximately accurate, and consistent boundary conditions are difficult to formulate [12].

The Maxwell solver presented here leads to the solution of Eq. (44) for boundary conditions (47) and (48), which correspond to perfectly conducting and magnetic mirror boundaries, respectively. It is shown that the solution of Maxwell's equations with these boundary conditions does not present spurious solutions and is unique.

Numerical results, including an electromagnetic wave in vacuo and an ion tearing mode (GEM challenge), demonstrate the accuracy of the solutions and good agreement with those provided by explicit PIC methods.

\section{ACKNOWLEDGMENTS}

The authors gratefully thank J. F. Drake and M. A. Shay for providing the simulation data used for the comparison in Fig. 8 and P. L. Pritchett for providing the simulation data represented in Fig. 9. This research is 
supported by the United States Department of Energy, under Contract W-7405-ENG-36 and by NASA, under the "Sun Earth Connection Theory Program." The supercomputer used in this investigation was provided by funding from JPL Institutional Computing and Information Services and the NASA Offices of Space Science and Earth Science.

\section{REFERENCES}

1. F. Assous, P. Degond, E. Heintze, P. A. Raviart, and J. Segre, On a finite-element method for solving the 3-dimensional Maxwell equations, J. Comput. Phys. 109, 222 (1993).

2. F. Assous, P. Ciarlet, and E. Sonnendrücker, Resolution of the Maxwell equations in a domain with reentrant corners, Model. Math. Anal. Numer. 32, 359 (1998).

3. D. C. Barnes, T. Kamimura, J.-N. Leboeuf, and T. Tajima, Implicit particle simulation of magnetized plasmas, J. Comput. Phys. 52, 480 (1983).

4. P. A. Bernhardt and J. U. Brackbill, Solution of elliptic equations using fast Poisson solvers, J. Comput. Phys. 53, 382 (1984).

5. T. S. Bird, Propagation and radiation of characteristics of rib waveguide, Electron. Lett. 13, 401 (1997).

6. C. K. Birdsall and A. B. Langdon, Plasma Physics via Computer Simulation (McGraw-Hill, New York, 1985).

7. J. Birn, J. F. Drake, M. A. Shay, B. N. Rogers, R. E. Denton, M. Hesse, M. Kuznetsova, A. W. Ma, A. Bhattacharjee, A. Otto, and P. L. Pritchett, Geospace Environment Modeling (GEM) magnetic reconnection challenge, J. Geophys. Res. 106, 3715 (2001).

8. D. Biskamp, E. Schwartz, and J. F. Drake, Two fluid theory of collisionless magnetic reconnection, Phys. Plasmas 4, 1002 (1997).

9. J. U. Brackbill and D. W. Forslund, An implicit method for electromagnetic plasma simulation in two dimensions, J. Comput. Phys. 46, 271 (1982).

10. J. U. Brackbill, D. W. Forslund, K. B. Quest, and D. Winske, Nonlinear evolution fo the lower-hybrid drift instability, Phys. Fluids 27, 2682 (1984).

11. J. U. Brackbill and D. W. Forslund, Simulation of low-frequency electromagnetic phenomena in plasmas, in Multiple Time Scales, edited by J. U. Brackbill and B. I. Cohen (Academic Press, San Diego, 1985), p. 271.

12. A. B. Langdon and D. C. Barnes, Direct implicit plasma simulation, in Multiple Time Scales, edited by J. U. Brackbill and B. I. Cohen (Academic Press, San Diego, 1985), p. 271.

13. J. U. Brackbill and H. X. Vu, Electron kinetic effects in switch-off slow shocks, Geophys. Res. Lett. 20, 2015 (1993).

14. B. I. Cohen, A. B. Langdon, D. W. Hewett, and R. J. Pocassini, Performance and optimization of direct implicit particle simulation, J. Comput. Phys. 81, 151 (1989).

15. B. I. Cohen, A. B. Langdon, and A. Friedman, Implicit time integration for plasma simulation, J. Comput. Phys. 46, 15 (1982).

16. J. Denavit, Time-filtering particle simulations with $\omega_{p e} \Delta t \gg 1$, J. Comput. Phys. 42, 337 (1981).

17. J. Dreher, U. Arendt, and K. Schindler, Particle simulations of collisionless reconnection in magnetotail configuration including electron dynamics, J. Geophys. Res. 101, 27375 (1996).

18. T. Zh. Esirkepov, Exact charge conservation scheme for particle-in-cell simulation with an arbitrary formfactor, Comput. Phys. Commun. 135, 144 (2001).

19. D. W. Forslund and J. U. Brackbill, Magnetic-field induced surface transport on laser-irradiated foils, Phys. Rev. Lett. 48, 1614 (1982).

20. D. W. Forslund, K. B. Quest, J. U. Brackbill, and K. Lee, Collisionless dissipation in quasi-perpendicular shocks, J. Geophys. Res. 89, 2142 (1984).

21. M. Hesse, J. Birn, and M. M. Kuznetsova, Collisionless magnetic reconnection: Electron processes and transport modelling, J. Geophys. Res. 106, 3721 (2001).

22. D. W. Hewett and A. B. Langdon, Electromagnetic direct implicit plasma simulation, J. Comput. Phys. 72 , 121 (1987).

23. D. W. Hewett, G. E. Frances, and C. E. Max, New regimes of magnetic reconnection in collisionless plasmas, Phys. Rev. Lett. 61, 893 (1988). 
24. P. Hillion, Beware of Maxwell's divergence equations, J. Comput. Phys. 132, 154 (1997).

25. R. W. Hockney and J. W. Eastwood, Computer Simulation Using Particles (Adam Hilger, Bristol and Philadelphia, 1988).

26. R. Horiuchi and T. Sato, Three-dimensional particle simulation of plasma instabilities and collisionless reconnection in a current sheet, Phys. Plasmas 6, 4565 (1999).

27. T. Kamimura, E. Montalvo, D. C. Barnes, J. N. Leboeuf, and T. Tajima, Implicit particle simulation of electromagnetic plasma phenomena, J. Comput. Phys. 100, 77 (1992).

28. U. Kangro and R. Nicolaides, Divergence boundary conditions for vector Helmholtz equations with divergence constraints, Model. Math. Anal. Numer. 33, 479 (1999).

29. D. A. Knoll, G. Lapenta, and J. U. Brackbill, A multilevel iterative field solver for implicit, kinetic, plasma simulation, J. Comput. Phys. 149, 377 (1999).

30. W. L. Kruer, The Physics of Laser Plasma Interactions (Addison-Wesley, Redwood City, CA, 1988).

31. M. M. Kuznetsova, M. Hesse, and D. Winske, Collisionless reconnection supported by nongyrotropic pressure effects in hybrid and particle simulations, J. Geophys. Res. 106, 3799 (2001).

32. B.-N. Jiang, J. Wu, and L. A. Povinelli, The origin of spurious solutions in computational electromagnetics, J. Comput. Phys. 125, 104 (1996).

33. A. B. Langdon and B. F. Lasinski, Electromagnetic and relativistic plasma simulation methods, in Methods in Computational Physics, edited by J. Killeen (Academic Press, New York, 1976), p. 327.

34. A. B. Langdon, B. I. Cohen, and A. Friedman, Direct implicit large time-step particle simulation of plasmas, J. Comput. Phys. 51, 107 (1983).

35. G. Lapenta and J. U. Brackbill, Contact discontinuities in collisionless plasmas: A comparison of hybrid and kinetic simulations, Geophys. Res. Lett. 23, 1713 (1996).

36. G. Lapenta and J. U. Brackbill, Implicit Adaptive Grid Plasma Simulation, 5th Int. School/Symposium for Space Simulation, Kyoto, Japan (March 13-19, 1997).

37. G. Lapenta and J. U. Brackbill, 3D reconnection due to oblique modes: A simulation of Harris current sheet, Nonlinear Proc. Geophys. 7, 151 (2000).

38. Deleted in proof.

39. B. Lembege and F. Simonet, Hybrid and particle simulations of an interface expansion and of collisionless shock: A comparative and qualitative study, Phys. Plasmas 8, 3967 (2001).

40. I. D. Mayergoyz and J. D'Angelo, A new point of view on the mathematical structure of Maxwell's equations, IEEE Trans. Magnetics 29, 1315 (1993).

41. R. J. Mason, Implicit moment particle simulation of plasmas, J. Comput. Phys. 41, 233 (1981).

42. R. J. Mason, An electromagnetic field algorithm for 2D implicit plasma simulation, J. Comput. Phys. 71, 429 (1987).

43. R. J. Mason, P. L. Auer, R. N. Sudan, B. V. Olivier, C. E. Seyler, and J. B. Greenly, Nonlinear magnetic field transport in opening switch plasmas, Phys. Fluids B 5, 1115 (1993).

44. M. Oieroset, T. D. Phan, M. Fujimoto, R. P. Lin, and R. P. Lepping, In situ detection of collisionless reconnection in the Earth's magnetotail, Nature 412, 414 (2001).

45. P. L. Pritchett, Geospace Environment Modeling magnetic reconnection challenge: Simulation with a full particle electromagnetic code, J. Geophys. Res. 106, 3783 (2001).

46. K. B. Quest, D. W. Forslund, J. U. Brackbill, and K. Lee, Collisionless dissipation processes in quasi-parallel shocks, Geophys. Res. Lett. 10, 471 (1983).

47. P. W. Rambo, J. Ambrosiano, A. Friednam, and D. E. Nielsen, Jr., Temporal and spatial filtering remedies for dispertion in electromagnetic particle codes, in Proc. 13th Conference on the Numerical Simulation of Plasmas, Santa Fe, New Mexico (September 17-20, 1989). [Paper PMA 9]

48. Y. Saad and M. H. Schultz, GMRES: A Generalised Minimal Residual Algorithm for Solving Nonsymmetric Linear Systems, SIAM J. Sci. Stat. Comput. 7, 856 (1986).

49. E. Schwieg and W. B. Bridges, Computer analysis of dielectric waveguide: A finite-difference method, IEEE Trans. Microwave Theory Technol. 32, 531 (1984).

50. J. S. Shang and D. Gaitonde, Characteristic-based, time-dependent Maxwell equation solvers on a general curvilinear frame, AIAA J. 33, 491 (1995). 
51. M. A. Shay, J. F. Drake, B. N. Rogers, and R. E. Denton, Alfvenic collisionless magnetic reconnection and the Hall term, J. Geophys. Res. 106, 3759 (2001).

52. H. Shimazu, S. Machida, and M. Tanaka, Macroparticle simulation of collisionless parallel shocks generated by solar wind and planetary plasma interactions, J. Geophys. Res. 101, 7647 (1997).

53. C. C. Su, Origin of spurious modes in the analysis of optical fiber using the finite-element or finite-difference technique, Electron. Lett. 21, 858 (1985).

54. D. Sulsky and J. U. Brackbill, A numerical method for suspension flow, J. Comput. Phys. 96, 339 (1991).

55. T. Tajima, Computational Plasma Physics: With Applications to Fusion and Astrophysics (Addison-Wesley, Redwood City, CA, 1989).

56. M. Tanaka, Macroscale implicit electromagnetic particle simulation of magnetized plasmas, J. Comput. Phys. 79, 209 (1988).

57. M. Tanaka, The Macro-EM particle simulation method and a study of collisionless reconnection, J. Comput. Phys. 87, 117 (1995).

58. J. Villasenor and O. Buneman, Rigorous charge conservation for local electromagnetic field solvers, Comput. Phys. Commun. 69, 306 (1992).

59. H. X. Vu and J. U. Brackbill, CELEST1D: An implicit, fully kinetic model for low-frequency, electromagnetic plasma simulation, Comput. Phys. Commun. 69, 253 (1992).

60. J. R. Winkler and J. B. Davies, Elimination of spurious modes in finite-element analysis, J. Comput. Phys. 56, 1 (1984).

61. D. Winske and N. Omidi, A nonspecialist's guide to kinetic simulations of space plasmas, J. Geophys. Res. 101, 17287 (1996).

62. M. A. Yates, D. B. VanHulsteyn, H. Rutkowski, G. Kyrala, and J. U. Brackbill, Experimental evidence for self-generated magnetic fields and remote energy deposition in laser-irradiated targets, Phys. Rev. Lett. 49, 1702 (1982). 